\title{
THE STRUCTURE OF THE MODULI SPACES OF CURVES AND ABELIAN VARIETIES
}

\author{
by DAVID MUMFORD
}

$\S 1$. The purpose of this talk is to collect together what seem to me to be the most basic moduli spaces (for curves and abelian varieties) and to indicate some of their most important interrelations and the key features of their internal structure, in particular those that come from the theta functions. We start with abelian varieties. Fix an integer $g \geqslant 1$. To classify $g$-dimensional abelian varieties, the natural moduli spaces are:

$$
\mathscr{A}^{(n)}=\left\{\begin{array}{l}
\text { moduli space of pairs }(X, \lambda), X \text { a } g \text {-dimensional } \\
\text { abelian variety, } \lambda: X \rightarrow \hat{X} \text { a polarization such } \\
\text { that } \operatorname{deg}(\lambda)=n^{2}
\end{array}\right\}
$$

Here and below when we talk of a moduli space, we mean a coarse moduli space in the sense of [11], p. 99 and in all cases these moduli spaces will actually exist as schemes of finite type over $\operatorname{Spec}(Z)$. This can be proven by the methods of [11], Ch. 7, for instance, which also shows that all the moduli spaces used are quasi-projective at least over every open set $\operatorname{Spec} Z\left[\frac{1}{p}\right]$.

The local structure of $\mathscr{A}^{(n)}$ seems quite difficult to work out at some points. However, for every sequence $\delta_{1}, \ldots, \delta_{\mathrm{g}}$ such that $\delta_{1}|\ldots| \delta_{\mathrm{g}}, \prod_{i=1}^{g} \delta_{i}=n$, let

$$
\mathscr{A}^{(\delta)}=\left\{\begin{array}{c}
\text { the open subscheme of } \mathscr{A}^{(n)} \text { of pairs }(X, \lambda) \\
\text { such that } \\
\operatorname{ker}(\lambda) \cong \prod_{1}^{g} Z / \delta_{i} Z \times \prod_{1}^{g} \mu_{\delta_{i}}
\end{array}\right\}
$$

The $\mathscr{A}^{(\delta)}$ 's are disjoint and exhaust all of $\mathscr{A}^{(n)}$ except for $(X, \lambda)$ 's such that char|n and ker $(\lambda)$ contains a subgroup isomorphic to $\alpha_{p}$. The local structure of $\mathscr{A}^{(\delta)}$ is not hard to work out (using results of Serre-Tate [20], and Grothendieck and myself on the formal deformation theory of abelian varieties and $p$-divisible groups, see Oort [17]). In particular all components of $\mathscr{A}^{(\delta)}$ dominate $\operatorname{Spec}(Z)$. Now I have proven that for all $n$ and all $p$, the open subset of $\mathscr{A}^{(n)} \times \operatorname{Spec} Z / p Z$ of $(X, \lambda)$ 's such that $X$ is ordinary $\left(^{*}\right)$ is dense (cf. [14] for a sketch of the proof). Therefore $\bigcup_{\delta} \mathscr{A}^{(\delta)}$ is dense in $\mathscr{A}^{(n)}$. Since $\mathscr{A}^{(\delta)} \times \operatorname{Spec}(C)$ is irreducible (see below), it follows that the components of $\mathscr{A}^{(n)}$ are the closures $\overline{\mathscr{A}}^{(\delta)}$ of the $\mathscr{A}^{(\delta)}$ and that all of them dominate $\operatorname{Spec}(Z)$. It is not

$\left(^{*}\right)$ i. e. has the maximal number $p^{g}$ of points of order $p$. 
known, however, whether the geometric fibres of $\mathscr{A}^{(\delta)}$ over finite primes are irreducible or not.

Now these various schemes $\mathscr{A}^{(n)}$ are all related by the isogeny correspondences:

$$
Z_{n_{1}, n_{2}, k}=\left\{\begin{array}{l|l}
(X, \lambda) \varepsilon \mathscr{A}^{\left(n_{1}\right)},(Y, \mu) \varepsilon \mathscr{A}^{\left(n_{2}\right)} & \begin{array}{c}
\exists \text { an isogeny } \pi: X \rightarrow Y \\
\text { of degree } k \text { such that } \\
n_{1}{ }^{2} \hat{\pi} \circ \mu \circ \pi=\left(k n_{2}\right)^{2} \lambda
\end{array}
\end{array}\right\}
$$

To uniformize all of these, one introduces a second more convenient sequence of moduli spaces. Firstly, over the base scheme $Z\left[\zeta_{n}\right], \zeta_{n}$ a primitive $n$-th root of 1 , let

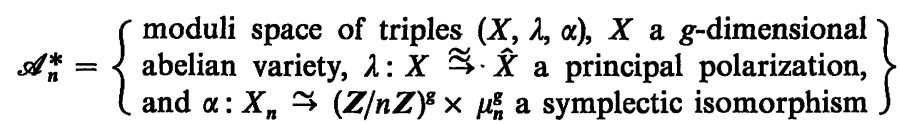

These spaces are normal and irreducible and form a tower with respect to the natural quasi-finite morphisms $\mathscr{A}_{n m}^{*} \rightarrow \mathscr{A}_{n}^{*}$ given by $(X, \lambda, \alpha) \mapsto\left(X, \lambda\right.$, res $\left.{ }_{X_{n}} \alpha\right)$. Secondly, we enlarge these schemes somewhat by letting $\mathscr{A}_{n}$ be the normalization of $\mathscr{A}_{1}$ in the field of rational functions $Q\left(\mathscr{A}_{n}^{*}, \zeta_{n}\right)$. Then $\mathscr{A}_{n}$ is a normal irreducible scheme in which $\mathscr{A}_{n}^{*}$ is an open subscheme, and the $\mathscr{A}_{n}$ 's form a tower with respect to finite morphisms $\mathscr{A}_{n m} \rightarrow \mathscr{A}_{n}$. Note that $\mathscr{A}_{n}=\mathscr{A}_{n}^{*}$ except over primes dividing $n$. Moreover, if $n \geqslant 3, \mathscr{A}_{n}$ is smooth over $Z$ except at non-ordinary abelian schemes in characteristics dividing $n$. Next, we can uniformize very nearly all of $\mathscr{A}^{(\delta)}$ by the natural morphism:

$$
\begin{aligned}
\mathscr{A}_{\delta_{g}}^{*} & \rightarrow \mathscr{A}^{(\delta)} \\
(X, \lambda, \alpha) & \mapsto(Y, \mu)
\end{aligned}
$$

where $Y$ is the etale covering of $X$ defined by requiring its dual to be the quotient:

$$
\hat{Y}=\hat{X} / \alpha^{-1}\left[(0) \times \Pi \mu_{\delta_{i}}\right],
$$

and $\mu$ is the polarization on $Y$ induced by $\lambda$. In the tower $\left\{\mathscr{A}_{n}\right\}$ one now has the Hecke ring of correspondences instead of the isogeny correspondences. These come essentially from 2 types of morphisms:

(a) $Q\left(\mathscr{A}_{n}, \zeta_{n}\right)$ is a Galois extension of $Q\left(\mathscr{A}_{1}, \zeta_{n}\right)$ with Galois group $\operatorname{Sp}(2 g, Z / n Z)$, hence $\operatorname{Sp}(2 g, Z / n Z)$ acts as a group of automorphisms of $\mathscr{A}_{n}$;

(b) the morphism

$$
\begin{aligned}
\mathscr{A}_{n}^{*} & \rightarrow \mathscr{A}_{1} \\
(X, \lambda, \alpha) & \mapsto(Y, \mu)
\end{aligned}
$$

(where $Y=X / \alpha^{-1}\left[(Z / n Z)^{g} \times(0)\right]$, and if $\pi: X \rightarrow Y$ is the natural map, then $\mu: Y \cong \hat{Y}$ is determined by the requirement $\hat{\pi} \circ \mu \circ \pi=n \lambda)$. For a discussion on Hecke operators in the classical case, see Shimura [21]. The picture is even clearer when you pass to an inverse limit: e. g. for all $n$,

where

$$
\lim _{\leftarrow} \mathscr{A}_{n^{k}} \times \operatorname{Spec} R_{n}
$$

$$
R_{n}=Z\left[\frac{1}{n}, \zeta_{n}, \zeta_{n^{2}}, \ldots\right]
$$

exists as a scheme and $\prod_{p \mid n} \operatorname{Sp}\left(2 g, Q_{p}\right)$ acts on it (See [12], $\S 9$ for the case $\left.n=2\right)$. 
Over the complex ground field, these moduli spaces have well-known analytic uniformizations coming from the theory of Siegel modular forms:

where

$$
\begin{aligned}
\mathscr{A}^{(n)} \times \operatorname{Spec}(C) & =\coprod_{\delta} \mathscr{A}^{(\delta)} \times \operatorname{Spec}(C) \\
\mathscr{A}^{(\delta)} \times \operatorname{Spec}(C) & =\mathfrak{T} / \Gamma_{\delta} \\
\mathscr{A}_{n} \times \operatorname{Spec}(C) & =\mathscr{A}_{n}^{*} \times \operatorname{Spec}(C)=\mathfrak{H} / \Gamma(n)
\end{aligned}
$$

where

$$
\begin{gathered}
\mathfrak{S}=\text { Siegel upper } \frac{1}{2}-\text { plane }=\left\{\begin{array}{l}
Z \begin{array}{l}
Z=g \times g \text { complex matrix } \\
{ }^{\prime} Z=Z, \operatorname{Im} Z>0
\end{array} \\
\Gamma(n)=\left\{A \in \operatorname{Sp}(2 g, Z) /( \pm I) \mid A=I_{2 \mathrm{~g}} \bmod n\right\}
\end{array}\right. \\
\Gamma_{\delta}=\left\{A \in G L(2 g, Z) /\left.( \pm I)\right|^{i} A . J_{\delta} . A=J_{\delta}\right\},
\end{gathered}
$$

$$
J_{\delta}=\left(\begin{array}{ccc|cc} 
& & & \delta_{1} & \\
& 0 & & \ddots & \\
& & & & \\
& & \delta_{g} \\
\hline-\delta_{1} & & & & \\
& & \ddots & & \\
& & \ddots & & \\
& & & &
\end{array}\right)
$$

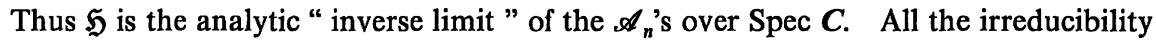
assertions made so far are proven by these analytic uniformizations.

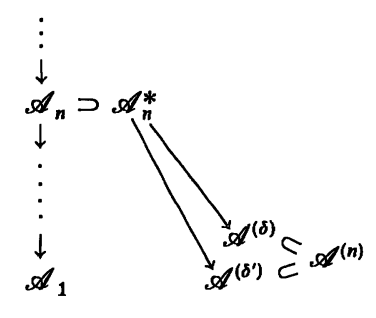

Summary of moduli spaces.

$\S 2$. The next point is that there is a moduli space intermediate in the tower between $\mathscr{A}_{n}^{*}$ and $\mathscr{A}_{2 n}^{*}$ on which there are canonical coordinates. Following Igusa, we christen this $\mathscr{A}_{n, 2 n}^{*}$ and it is defined as follows in char. $\neq 2$ :

$$
\mathscr{A}_{n, 2 n}^{*}=\left\{\begin{array}{l}
\text { moduli space of triples }(X, L, \alpha), X \text { an abelian } \\
\text { variety of dimension } g, L \text { an ample symmetric } \\
\text { invertible sheaf, } \alpha \text { a symmetric isomorphism: } \\
\quad \alpha: \mathscr{G}(L) \simeq G_{m} \times(Z / n Z)^{\mathrm{g}} \times \mu_{n}^{\mathrm{g}} \\
\text { such that } \\
\text { i) if } n \text { even, } e_{*}^{L} \equiv 1 \text { on } X_{2}, \\
\text { ii) if } n \text { odd, } e_{*}^{L} \text { takes the value }+1 \text { more often } \\
\text { than the value }-1 .
\end{array}\right\}
$$


For definitions of $\mathscr{G}(L), e_{*}^{L}$, etc., see [12], $\S 1,2$ and [15], $\S 23$. There is an obvious map

$$
\begin{aligned}
\mathscr{A}_{n, 2 n}^{*} & \rightarrow \mathscr{A}_{n}^{*} \\
(X, L, \alpha) & \mapsto\left(X, \frac{1}{n} \varphi_{L}, \bar{\alpha}\right)
\end{aligned}
$$

where $\bar{\alpha}$ is the induced map from $\mathscr{G}(L) / G_{m} \cong X_{n}$ to $(Z / n Z)^{g} \times \mu_{n}^{g}$. There is a not so obvious map $\mathscr{A}_{2 n}^{*} \times \operatorname{Spec} Z\left[\frac{1}{2}\right] \rightarrow \mathscr{A}_{n, 2 n}^{*}$ (see [12], § 2). Over $C, \mathscr{A}_{n, 2 n}^{*}$ is simply the quotient $\mathfrak{T} / \Gamma(n, 2 n)$, where $\Gamma(n, 2 n)$ is the subgroup between $\Gamma(n)$ and $\Gamma(2 n)$ described by Igusa [9]. Canonical coordinates on $\mathscr{A}_{n, 2 n}^{*}$ (where $n \geqslant 2$ ) are defined as follows:

i) $\mathscr{G}(L)$ and hence $G_{m} \times(Z / n Z)^{g} \times \mu_{n}^{g}$ acts on $H^{0}(X, L)$. Write this action as $U_{(\lambda, a, b)}: H^{0}(X, L) \rightarrow H^{0}(X, L)$,

ii) there is a section $\sigma \in H^{0}(X, L)$ unique up to scalars such that $U_{(1,0, c)} \sigma=\sigma$, all $c \in \mu_{n}^{g}$,

iii) let $\sigma \rightarrow \sigma(0)$ denote evaluation of sections at $0 \in X$. We obtain a function:

$$
\begin{aligned}
(Z / n Z)^{g} & \rightarrow K \\
\alpha & \mapsto\left(U_{(1, \alpha, 0)} \sigma\right)(0)
\end{aligned}
$$

unique up to multiplication by a constant, which is never identically zero.

iv) If $N=n^{g}-1$, and the homogeneous coordinates of $\boldsymbol{P}_{N}$ are put in one-one correspondence with the elements of $(Z / n Z)^{g}$, this defines a morphism:

$$
\Theta: \mathscr{A}_{n, 2 n}^{*} \rightarrow \boldsymbol{P}_{N}
$$

THEOREM. - If $n \geqslant 4, \Theta$ is an immersion.

This theorem was proven over $\boldsymbol{C}$ for various $n$ 's by Baily [4] and Igusa [9]; in the general case, all the essentials for the proof are in [13]. Over $C, \Theta$ is the morphism defined by

$$
Z \mapsto\left(\ldots, \theta_{n Z}\left[\begin{array}{c}
0 \\
\alpha / n
\end{array}\right](0), \ldots\right)_{\alpha \in(Z / n Z)^{g}}
$$

where $Z \in \mathfrak{H}$, and $\theta$ is Riemann's theta function. If $8 \mid n$, one can even find a finite set of homogeneous quartic polynominals-Riemann's theta relations-such that the image of $\Theta$ is an open part of the subscheme of $\boldsymbol{P}_{N}$ defined by these quartics (see [12], § 6).

Even in the char. $p$ case, it is possible to reformulate these canonical coordinates as values of a type of theta function. These theta functions are not functions on the universal covering space of $X$, but rather on the Tate group.

If $p=$ char. of ground field,

$V(X)=$ group of sequences $\left\{x_{i}\right\}, i \geqslant 1$ but $p \nmid i$, where $x_{i} \in X, n x_{i n}=x_{i}$ and $x_{1}$ has finite order $k$ prime to $p$.

Let $T(X)=\left\{\left(x_{i}\right) \in V(X)\right.$ such that $\left.x_{1}=0\right\}$.

We get an exact sequence:

$$
0 \rightarrow T(X) \rightarrow V(X) \rightarrow \text { (torsion on } X \text { prime to } p) \rightarrow 0
$$


We use the result:

THeorem. - Let $L$ be an ample symmetric invertible sheaf of degree 1 on an abelian variety $X$ of char. p. If $p \nmid 2 n$, then for all $x \in X_{n}$ for every choice of a point $y \in X$ such that $2 n y=x$, there is a canonical isomorphism:

$$
L \otimes_{\theta_{X}} k(x) \cong L \otimes_{\theta_{X}} k(0)
$$

Corollary. - If $\sigma \in \Gamma(L)$, then evaluating $\sigma$ via the above isomorphisms defines a function

$$
\theta: V(X) \rightarrow L \otimes_{\theta_{X}} k(0) \cong k
$$

such that if $x \in \frac{1}{n} T(X)$, then $\theta(x+y)=\theta(x)$ if $y \in 2 n T(X)$.

In fact the functions that we obtain in this way have the following properties:

a)

$$
\theta(x+a)=e_{*}\left(\frac{a}{2}\right) e\left(\frac{a}{2}, x\right) \theta(x), x \in V(X), a \in T(X)
$$

where

$$
e_{*}: \frac{1}{2} T(X) \rightarrow\{ \pm 1\} \quad \text { and } \quad e: V(X) \times V(X) \rightarrow k^{*}
$$

are the functions induced by $e_{*}^{L}$ and $e_{n}$ on $V(X)$.

b) $\theta(-x)= \pm \theta(x)$, the sign depending on the Arf invariant of $e_{*}^{L}$.

where

$$
\prod_{i=1}^{4} \theta\left(x_{i}\right)=2^{-g} \sum_{\eta \in \frac{1}{2} T(X) / T(X)} e(y, \eta)\left(\prod_{i=1}^{4} \theta\left(x_{i}+y+\eta\right)\right)
$$

$$
y=-\frac{1}{2} \Sigma x_{i}
$$

d) $\quad \forall x \in V(X), \quad \exists \eta \in \frac{1}{2} T(X) \quad$ such that $\quad \theta(x+\eta) \neq 0$.

e) Up to an elementary linear transformation whose coefficients are roots of 1 , the set of values of $\theta$ on $\frac{1}{n} T(X)$ is equal to the set of values of the canonical coordinates $\Theta$ on the triple $\left(X, L^{n^{2}}, \alpha\right)$ (for any symmetric $\alpha$ ).

$f$ ) Over $C$, if $Z$ is a period matrix for $X, \theta$ is essentially the function $a \mapsto \theta_{Z}[a](0)$, $a \in Q^{2 g}$.

g) Moreover, if we restrict the domain to $V_{2}(X)$, this 2-adic Tate group, all functions $\psi: V_{2}(X) \rightarrow k$ satisfying $\left.\left.\left.a\right), b\right), c\right)$ and $d$ ) arise from a unique principally polarized abelian variety.

(Cf. [12], § 8 through $\S 12$ ).

$\S 3$. We turn next to curves. Fix $g \geqslant 2$. Let

$$
\mathscr{M}=\left\{\begin{array}{l}
\text { moduli space of non-singular } \\
\text { complete curves } C \text { of genus } g
\end{array}\right\}
$$


$\mathscr{M}$ is not only irreducible, but it has irreducible geometric fibres over $\operatorname{Spec}(Z)$, cf. [5]. This is proven by introducing a compacification $\overline{\mathscr{M}}$ of $\mathscr{M}$, where

$$
\overline{\mathscr{M}}=\left\{\begin{array}{l}
\text { moduli space of stable complete curves } C \\
\text { such that } \operatorname{dim} H^{1}\left(o_{C}\right)=g
\end{array}\right\}
$$

and where a stable curve is one with at most ordinary double points and such that every non-singular rational component has at least 3 double points on it.

$\overline{\mathscr{M}}$ has recently been proven by $\mathrm{F}$. Knudsen, Seshadri and my self to be a scheme projective over $\boldsymbol{Z}$

Define:

$$
\begin{aligned}
t: \mathscr{M} & \rightarrow \mathscr{A}_{1} \\
C & \mapsto\left(\operatorname{Pic}^{0}(C), \lambda\right)
\end{aligned}
$$

where $\lambda$ is the theta polarization, viz: fixing a base point $x_{0}$ on $C$, we obtain a morphism:

hence

$$
\begin{aligned}
\phi: C & \rightarrow \text { Pic }^{0} C \\
x & \mapsto \text { class of } o_{C}\left(x-x_{0}\right)
\end{aligned}
$$

$$
\widehat{\operatorname{Pic}}^{0} C=\operatorname{Pic}^{0}\left(\mathrm{Pic}^{0} C\right) \stackrel{\phi^{*}}{\rightarrow} \operatorname{Pic}^{0}(C)
$$

and $\lambda=-\left(\phi^{*}\right)^{-1}$.

According to Torelli's theorem (cf. [1], [10]), $t$ is injective on geometric points. Its image however is not closed since $t$ extends to a morphism on $\tilde{\mathscr{M}}$ :

$$
\left\{\begin{array}{l}
\text { Stable curves made from } \\
\text { non-singular components } \\
\text { connected together like a } \\
\text { tree }
\end{array}\right\}=\begin{aligned}
& \overline{\mathscr{M}} \\
& \widetilde{\mathscr{M}} \\
& \cup
\end{aligned}
$$

and $\tilde{t}$ can be shown to be a proper morphism taking each stable curve $C$ in $\tilde{\mathscr{M}}$ to $\mathrm{Pic}^{0} C$ with a suitable polarization (cf. Hoyt [8]). Let $\mathscr{T}=\overline{t(\mathscr{M})}=\tilde{t}(\tilde{\mathscr{M}})$ : this is called the Torelli locus. A famous classical problem is to describe $\mathscr{T}$, or its inverse image in some $\mathscr{A}_{n}$, by explicit equations, e. g. polynomials in the theta-nulls. Partial results on this were obtained in characteristic zero by Riemann [18], Schottky, and SchottkyJung [19]. Their results have been rigorously established recently by Farkas and Rauch [6], and some interesting generalizations have been given by Fay [7]. A completely different approach to this problem is given in the beautiful paper of Andreotti and Mayer [2]. I want to finish by sketching the key point in Schottky's theory and stating a theorem on what his equations do characterize. We assume char. $\neq 2$.

Let $\Pi: \hat{C} \rightarrow C$ be an etale double covering, and let $\imath: \hat{C} \rightarrow \hat{C}$ be the corresponding involution. If $g=$ genus of $C$, then $2 g-1=$ genus of $\hat{C}$. The Jacobians $J=\operatorname{Pic}^{0} C$, $\hat{J}=\mathrm{Pic}^{0} \hat{C}$ are related by 2 homeomorphisms:

$$
\hat{J} \underset{\Pi^{*}}{\stackrel{N m}{\rightleftarrows}} J
$$

such that $N m \circ \Pi^{*}=2_{J} . \quad l$ acts on $\hat{J}$ also. Define:

$$
P=\text { locus of points }\{l x-x\} \text { in } \hat{J} \text {. }
$$


We get isogenies:

$$
\begin{aligned}
\hat{J} & \stackrel{\beta}{\stackrel{\beta}{\rightleftarrows}} J \times P \\
\alpha(x, y) & =\Pi^{*} x+y \\
\beta(z) & =(N m z, z-\imath z)
\end{aligned}
$$

such that $\alpha \circ \beta=\beta \circ \alpha=$ mult. by 2. Next fix $\gamma$, a division class on $C$ such that $2 \gamma \equiv K_{C}$, the canonical divisor class, and such that $\operatorname{dim} H^{0}(o(\gamma)$ ) is even (cf. [16] for this). We get symmetric divisors:

$$
\begin{aligned}
& \Theta=\left\{\text { locus of div. classes } \sum_{1}^{8-1} P_{i}-\gamma\right\} \subset J \\
& \hat{\Theta}=\left\{\text { locus of div. classes } \sum_{1}^{2 g-2} P_{i}-\Pi^{*} \gamma\right\} \subset \hat{J}
\end{aligned}
$$

representing the standard polarizations of $J$ and $\hat{J}$.

\section{LEMMA}

a) $\Pi^{*-1}(\hat{\Theta})=\Theta+\Theta_{\kappa}$, where $\{0, \kappa\}=\operatorname{Ker}\left(\Pi^{*}\right)$.

b) $\exists$ a symmetric divisor $\Xi$ on $P$ such that $\hat{\Theta} . P=2 \Xi$.

c) $\alpha^{-1}(\Theta) \equiv \Theta \times P+\Theta_{\kappa} \times P+2 J \times \Xi$.

In particular, $\Xi$ has degree 1 and defines a principal polarization on $P$. Abstractly put now we have a situation with.

i) 3 abelian varieties $X, Y, Z$ of dimensions $g, g-1,2 g-1$ resp.,

ii) 3 ample degree 1 symmetric divisors $\Theta_{X} \subset X, \Theta_{Y} \subset Y, \Theta_{Z} \subset Z$, which define as in $\S 2$ theta-functions $\theta_{X}$ on $V(X), \theta_{Y}$ on $V(Y)$ and $\theta_{Z}$ on $V(Z)$,

iii) isogenies $Z \underset{\alpha}{\stackrel{\beta}{\rightleftarrows}} X \times Y$ such that $\alpha \circ \beta=\beta \circ \alpha=$ mult. by 2 . In such a case, $Z \cong X \times Y / H$, where $H$ is a so-called Göpel group, and $V(Z) \cong V(X) \times V(Y)$. Moreover $\theta_{Z}$ can be computed from $\theta_{X}$ and $\theta_{Y}$ by one of the basic theta formulas. But then the lemma, esp. part $a$ ), implies non-trivial identities on $\theta_{X}$ and $\theta_{Y}$. In fact, it follows that for a suitable $\eta \in \frac{1}{2} T(X)$ with image $\kappa$ in $X$ and a suitable homeomorphism:

$$
\begin{gathered}
\varphi:\left\{x \in \frac{1}{2} T(X) \mid e(x, \eta)=1\right\} \rightarrow \frac{1}{2} T(Y) \\
\left\{\begin{array}{l}
\frac{\theta_{X}(x) \cdot \theta_{X}(x+\eta)}{\theta_{Y}(\varphi x)^{2}}=\frac{\theta_{X}(y) \cdot \theta_{X}(y+\eta)}{\theta_{Y}(\varphi y)^{2}} \\
\text { all } \quad x, y \in \frac{1}{2} T(X) \\
\text { with } \quad e(x, \eta)=e(y, \eta)=1
\end{array}\right.
\end{gathered}
$$

If we globalize this set-up, we get the following moduli situation: $\mathscr{M}_{*}$ is to be the normalization of $\mathscr{M}$ is a suitable finite algebraic extension of its function field such that for every point of $\mathscr{M}_{*}$ there is given rationally not only a curve $C$ of genus $g$, but 
(a) a $(4,8)$-structure on $J$ (i. e. a point of $\mathscr{A}_{(4,8)}$ lying over $J$ in $\left.\mathscr{A}_{1}\right),(b)$ a double covering $\Pi: \hat{C} \rightarrow C,(c)$ a (4,8)-structure on $P$ (cf. [5], pp. 104-108 for a precise discussion of such " non-abelian levels "). Thus, if we let $\mathscr{A}$ 's (resp. $\mathscr{B}$ 's) represent moduli spaces for abelian varieties of dim. $g$ (resp. dim. $g-1$ ), we have morphisms:

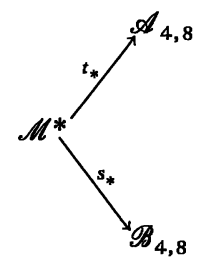

and since $\theta_{J}$ on $\frac{1}{2} T(J)\left(\right.$ resp. $\theta_{P}$ on $\left.\frac{1}{2} T(P)\right)$ are coordinates on $\mathscr{A}_{4,8}$ (resp. $\mathscr{B}_{4,8}$ ), the identities $\left(^{*}\right)$ define a locus $\mathscr{C} \subset \mathscr{A}_{4,8} \times \mathscr{B}_{4,8}$ (the $\eta$ and $\varphi$ must be independent of the curve you start with). We find:

TheOREM. - Im $\left(t_{*}, s_{*}\right)$ is an open subset of one of the components of locus $\mathscr{C}$ of solutions of the Schottky-Jung identites $\left(^{*}\right)$ inside the moduli space $\mathscr{A}_{4,8} \times \mathscr{B}_{4,8}$.

\section{REFERENCES}

[1] A. ANdreottr. - On a theorem of Torelli, Am. J. Math., 80 (1958).

[2] - and A. MAYER. - On period relations for abelian integrals on algebraic curves, Annali di Scuolo Norm. di Pisa (1967).

[3] M. Artin. - The implicit function theorem in algebraic geometry, in Algebraic Geometry, Oxford Univ. Press (1969).

[4] W. BaILY. - On the moduli of abelian varieties with multiplications, J. Math. Soc. Japan, 15 (1963).

[5] P. Deligne and D. Mumford. - The irreducibility of the space of curves of given genus, Pub. I. H. E. S., 36 (1969).

[6] H. Farkas and RaUch. - Two kinds of theta constants and period relations on a Riemann Surface, Proc. Nat. Acad. U. S., 62 (1969).

[7] J. FAY. - Special moduli and theta relations, Ph. D. Thesis, Harvard (1970).

[8] W. Hoyt. - On products and algebraic families of jacobian varieties, Annals of Math., 77 (1963).

[9] J. I. IguSA. - On the graded ring of theta-constants, Am. J. Math., 86 (1964) and 88 (1966).

[10] T. Matsusaka. - On a theorem of Torelli, Am. J. Math., 80 (1958).

[11] D. MUMFORD. - Geometric Invariant theory, Springer-Verlag, Heidelberg (1965).

[12] - . - On the equations defining abelian varieties, Inv. Math., Part I: vol. 1 (1966), Parts II and III: vol. 3 (1967).

[13] - - - Varieties defined by quadratic equations, in Questioni sulle varieta algebriche, Corsi dal C. I. M. E., 1969, Edizioni Cremonese, Roma.

[14] - . - Bi-extensions of formal groups, in Algebraic Geometry, Oxford Univ. Press (1969).

[15] -. - Abelian varieties, Oxford Univ. Press (1970). 
[16] - - - Theta characteristics of an algebraic curve, to appear in Annales de l'École Normale Supérieure (1971).

[17] F. OORT. - Finite group schemes, local moduli for abelian varieties and lifting problems, to appear in Proc. Nordic Summer school of 1970, Wolters-Noordhoff, Groningen.

[18] B. Riemann. - Collected works, Nachtrag IV, Dover edition (1953).

[19] F. Sснотткy and H. JUNG. - Neue Sätze über symmetralfunctionen und die Abelschen funktionen der Riemann'sche theorie, Sitzungber, Berlin Akad. Wissensch., vol. I (1909).

[20] J. P. SERre and J. TATE. - Proc. of seminar on formal groups at Woods Hole Summer Institute (1964), mimeographed notes.

[21] G. Shimura. - Moduli of abelian varieties and number theory, in algebraic groups and discontinuous subgroups, Am. Math. Soc. (1966).

Harvard University,

Department of Mathematics,

2, Divinity Avenue,

Cambridge, Mass. 02138

(U. S. A.) 
